University of Nebraska - Lincoln

DigitalCommons@University of Nebraska - Lincoln

Faculty Publications, Department of Physics and Astronomy

Research Papers in Physics and Astronomy

$5-9-2002$

\title{
Threshold-related effects in high-order harmonic generation
}

Bogdan Borca

Institute for Space Sciences, Bucharest, Romania

Anthony F. Starace

University of Nebraska-Lincoln, astarace1@unl.edu

A. V. Flegel

Voronezh State University, Voronezh, Russia

M. V. Frolov

Voronezh State University, Voronezh, Russia

N. L. Manakov

Voronezh State University, manakov@phys.vsu.ru

Follow this and additional works at: https://digitalcommons.unl.edu/physicsfacpub

Part of the Physics Commons

Borca, Bogdan; Starace, Anthony F.; Flegel, A. V.; Frolov, M. V.; and Manakov, N. L., "Threshold-related effects in high-order harmonic generation" (2002). Faculty Publications, Department of Physics and Astronomy. 49.

https://digitalcommons.unl.edu/physicsfacpub/49

This Article is brought to you for free and open access by the Research Papers in Physics and Astronomy at DigitalCommons@University of Nebraska - Lincoln. It has been accepted for inclusion in Faculty Publications, Department of Physics and Astronomy by an authorized administrator of DigitalCommons@University of Nebraska Lincoln. 


\title{
Threshold-related effects in high-order harmonic generation
}

\author{
Bogdan Borca* and Anthony F. Starace \\ Department of Physics and Astronomy, The University of Nebraska, Lincoln, Nebraska 68588-0111
}

A. V. Flegel, M. V. Frolov, and N. L. Manakov

Research and Education Center, Voronezh State University, 394693 Voronezh, Russia

(Received 5 December 2001; published 9 May 2002)

\begin{abstract}
We present exact $a b$ initio quantum results for harmonic generation (HG) by a weakly bound electron interacting with a strong monochromatic laser field. The analytic structure of the HG amplitude resulting from the multiphoton detachment thresholds is demonstrated. Our numerical results show that the HG spectrum is sensitive primarily to the distance in energy from the thresholds, thereby opening new possibilities for control of HG.
\end{abstract}

DOI: 10.1103/PhysRevA.65.051402

PACS number(s): $32.80 . \mathrm{Rm}, 42.65 . \mathrm{Ky}, 42.50 . \mathrm{Hz}$

Above threshold ionization (ATI) and high-order harmonic generation (HG) are two of the most well-studied processes in strong-field physics owing to the insight they provide on the interaction of intense laser light with atoms [1]. Because they both involve laser excitation of a bound electron, numerous studies have focused on their precise relationship (e.g., Ref. [2]). Also, because HG holds great promise for producing coherent short-wavelength radiation, efforts have been directed to enhancing HG intensities, such as by multiphoton resonance enhancement (e.g., Refs. [3,4]). Resonant structures in the intensity dependence of $\mathrm{HG}$ rates for the hydrogen atom were found in nonperturbative numerical calculations [5]. Well-known cusp like features [6] in the intensity dependence of particular high harmonics in the vicinity of multiphoton detachment thresholds have been predicted numerically [7] using a Keldysh approximation (KA) [8] for the three-dimensional zero-range potential (ZRP) model. Another theoretical study, however, has attributed such structures to constructive interference of intermediate-state ATI amplitudes and not to threshold effects [9], which is consistent with the model that the HG plateau results from constructive interference of quasiclassical electron orbits that return to the nucleus and emit a harmonic photon (see Ref. [10], and references therein). Recently, an $\mathrm{HG}$ experiment for Ar has found a resonant enhancement of the 13th harmonic in a narrow range of laser intensity [11]. Significantly, Ref. [11] employs a shaped laser focus, making it possible to observe atomic features in the HG spectrum. (Usually such features are difficult to observe experimentally owing to the intensity averaging over the laser focal region [3]). Although the enhancement is attributed to a 13 photon resonance with the $5 g$ state of $\operatorname{Ar}$ [11], it occurs in an intensity region in which ATI channels are closing.

In this Rapid Communication we analyze channel closing (CC) effects on HG for the case of a ZRP using an essentially exact, nonperturbative theoretical approach, the quasistationary quasienergy state (QQES) theory [12]. Our ab initio ap-

\footnotetext{
*On leave from the Institute for Space Sciences, BucharestMagurele 76900, Romania.
}

proach does not employ approximations common in analytic theories of HG $[7,9,10]$. Indeed, we show analytically the connection of our exact theory to other approaches when such approximations are made. Our analytic results reveal the expected threshold branch points [6], confirming the approximate numerical results of Ref. [7]. Our numerical results show not only that threshold anomalies (TAs) occur over a large range of harmonics in the plateau region, but also that the structure of the HG spectrum depends largely on the distance in energy from the ATI thresholds. In addition, the TAs produce significant enhancement of the HG spectrum, thereby holding forth the promise of providing a means to control HG intensities.

The QQES theory for a ZRP model has been discussed in detail elsewhere $[13,14]$. Its key quantities are: the quasienergy $\epsilon=\operatorname{Re} \epsilon-i \Gamma / 2$, where $\operatorname{Re} \epsilon$ and $\Gamma$ give the position and the width (decay rate) of the initial level $E_{0}$ in a laser field; and the Fourier coefficients $\phi_{\nu}$ of the periodic QQES wave function $\Phi_{\epsilon}(\mathbf{r}, t)$ for $r \rightarrow 0$ [13]. The laser field is given by $\mathbf{F}(t)=F \operatorname{Re}[\mathbf{e} \exp (-i \omega t)]$, where $F$ is the amplitude, $\omega$ is the frequency, and $\mathbf{e}$ is the polarization unit vector (generally complex for an elliptic polarization), e. $\mathbf{e}^{*}=1$. We employ scaled units, in which energies and $\hbar \omega$ are in units of $\left|E_{0}\right|$, and $F$ is in units of $F_{0}=\sqrt{2 m\left|E_{0}\right|^{3}} / e \hbar$. Within the QQES formalism, the amplitude $A_{n \omega}\left(\mathbf{e}^{\prime}\right)$ describing dipole emission of the $n$th harmonic photon with polarization $\mathbf{e}^{\prime}$ is [15]

$$
A_{n \omega}\left(\mathbf{e}^{\prime}\right)=\mathbf{e}^{\prime *} \cdot \widetilde{\mathbf{d}}_{n}=\frac{\omega}{\pi} \int_{0}^{2 \pi / \omega} d t\left\langle\widetilde{\Phi}_{\epsilon}\left|\mathbf{e}^{\prime *} \cdot \mathbf{r} e^{i n \omega t}\right| \Phi_{\epsilon}\right\rangle
$$

where use of the dual state $\tilde{\Phi}_{\epsilon}(\mathbf{r}, t)$ [for linear polarization, $\left.\widetilde{\Phi}_{\epsilon}(\mathbf{r}, t)=\Phi_{\epsilon}^{*}(\mathbf{r},-t)\right]$ as the bra vector gives the correct quantum definition of the HG amplitude in the QQES approach $[14,15]$. The total rate for the $n$th harmonic is proportional to $\left|\widetilde{\mathbf{d}}_{n}\right|^{2}$, where $\widetilde{\mathbf{d}}_{n}=\chi_{1} \mathbf{e}+\chi_{2} \mathbf{e}^{*}$ is expressed in terms of the susceptibilities $\chi_{1,2}$ [15].

Exact expressions for $\chi_{1,2}$ can be written in terms of the coefficients $\phi_{\nu}$ and the quasienergy $\epsilon$. By introducing two characteristic parameters of the problem, $\eta=\left(U_{p}-\epsilon\right) / \omega$ and $\xi=\left(2 U_{p}\right) / \omega$, where $U_{p}=F^{2} /\left(2 \omega^{2}\right)$ is the ponderomotive 
potential, one can express $\chi_{1}$ (and $\chi_{2}$ ) as an absolutely convergent series expansion in $\xi$ that shows explicitly its branch-point structure:

$$
\chi_{1}=\frac{2 \sqrt{\xi}}{n^{2} \omega} \sum_{\mu, \nu}^{\infty} \frac{(-\ell)^{|\nu|}}{|\nu| !} \widetilde{\phi}_{2(\mu+\nu)+n-1}^{*} \phi_{2 \mu} \chi_{1}^{(\mu, \nu)}
$$

where

$$
\begin{aligned}
\chi_{1}^{(\mu, \nu)}= & \sum_{m=|\nu|}^{\infty} \xi^{m} \frac{{ }_{2} F_{1}\left(\frac{|\nu|-m}{2}, \frac{1+|\nu|-m}{2} ;|\nu|+1 ; \ell^{2}\right)}{(m-|\nu|) !} \\
& \times\left[\frac{n}{2(n-1)}\left[\mathcal{D}_{m}^{\prime}\left(\eta_{\mu \nu}\right)-\mathcal{D}_{m}^{\prime}\left(\eta_{\mu \nu}+n-1\right)\right]\right. \\
& -\mathcal{D}_{m}\left(\eta_{\mu \nu}\right)-\mathcal{D}_{m}\left(\eta_{\mu \nu}+n-1\right)+\mathcal{D}_{m}\left(\eta_{\mu \nu}+n\right) \\
& \left.+\mathcal{D}_{m}\left(\eta_{\mu \nu}-1\right)\right]
\end{aligned}
$$

and

$$
\mathcal{D}_{m}(\eta)=\frac{2}{(2 m+3) ! !} \sum_{k=-m}^{m}(-1)^{k} C_{2 m}^{m+k}(\eta+k)^{m+3 / 2}
$$

In Eq. (3), ${ }_{2} F_{1}(-k, b ; c ; x)$ is a hypergeometric Gaussian polynomial involving the degree of linear polarization, $\ell$ $=\mathbf{e} \cdot \mathbf{e}$, of the laser field, $0 \leqslant \ell \leqslant 1$. Also, $\mathcal{D}_{m}^{\prime}(\alpha)$ $=(\partial / \partial \alpha) \mathcal{D}_{m}(\alpha)$ and the arguments of $\mathcal{D}_{m}$ involve $\eta_{\mu \nu}=\eta$ $+2 \mu+\nu$. In Eq. (4) $C_{a}^{b}$ is a binomial coefficient. The coefficients $\phi_{\nu}$ are normalized by the condition $\phi_{\nu}=\delta_{\nu, 0}$ at $F$ $=0$, and the branches of the square roots in Eq. (4) are chosen such that $\operatorname{Re} \sqrt{\eta+n}>0$ for $\operatorname{Re}(\eta+n)>0$ and $\operatorname{Im} \sqrt{\eta+n}<0$ for $\operatorname{Re}(\eta+n)<0$. The expression for $\chi_{2}$ (not shown) is similar to that for $\chi_{1}$; note, however, that only $\chi_{1}$ is nonzero in the limit of lowest-order perturbation theory (LOPT).

Using numerically calculated $\epsilon$ and $\phi_{\nu}[16]$, the analytic expressions for $\chi_{1,2}$ permit an essentially exact analysis of HG for arbitrary $F, \omega$, and $\ell$. In particular, from the exact Eqs. (2)-(4) easily follow all known HG rate expressions commonly used in approximate semianalytical analyses of the HG problem (most of which use a saddle-point analysis in terms of electron trajectories). Equations (2)-(4) for $\chi_{1}$ have the same form in the KA [8] (in which effects of the binding potential on the active electron are neglected except in the initial bound state); however, the coefficients $\phi_{\nu}$ reduce to the Bessel functions,

$$
\phi_{2 s}=(-1)^{s} J_{s}(\ell \xi / 4),
$$

and $\epsilon$ reduces to $E_{0}$, so that $\eta=\left(U_{p}+\left|E_{0}\right|\right) / \omega$. This KA limit of our exact results coincides with the " $S$-matrix approach" result [17], provided the latter includes the so-called continuum-continuum terms. Omitting these terms, as in Ref. [18], is equivalent to setting $\phi_{2 \mu}=\delta_{\mu 0}$ in Eq. (2). A third approximation is to keep only terms with $\mu=0$ and $\nu \leqslant 0$ in the KA limit of Eq. (2), which implies that only electron transitions involving absorption of $|\nu|$ photons are treated, as in Refs. $[9,19]$. Finally, our formulation differs from those of Refs. $[7,18,20]$ by our use of the dual function in Eq. (1). In the KA limit, in which the calculations in Refs. [7,20] were performed, our numerically exact results differ for only a few of the lowest $n$ harmonics (in particular, the first harmonic is not divergent). Beyond the KA, however, in general, any HG amplitude not employing the dual function (e.g., Ref. [20]) is divergent [15].

The series expansion (3) allows one to analyze $\chi_{1}$ explicitly as analytic function of $\omega$ and $F$. When $F$ and $\omega$ satisfy Re $\eta(F, \omega)=q$, where $q$ is an integer, the HG amplitude has a branch point near each $q$-photon ATI threshold. The ATI and HG channels are alternative final states of the problem, with the distinction that all HG channels are formally open for any $F$ and $\omega$, whereas ATI channels have thresholds. According to general multichannel reaction theory, the closing of the $q$ th ATI channel affects (through channel coupling) the open ATI (with $n>q$ ) and HG channels. For a shortrange potential, these TAs were predicted by Baz' [6]. In brief, the dominant contribution in a very small region around the threshold of the $q$ th ATI channel stems from the subchannel having the lowest photoelectron orbital angular momentum $L$; $s$ for even $q$ and $p$ for odd $q$ (for polarizations $\ell>0)$. In particular, for an $s$-closing channel there are four possible TAs; two cusps and two "steps" [6] resulting from the singular derivative of the $\mathrm{HG}$ amplitude with respect to $\eta$. In general, an $L$-angular-momentum $C C$ produces a singular derivative of order $L+1$ in the HG (and open ATI) rates.

Equations (2) and (3) demonstrate explicitly how the ATI CCs appear in the HG amplitude and how near any threshold $q$, a particular subset of terms in the series, those involving $(\eta-q)^{L+1 / 2}$, produce the resonantlike behavior of HG rates near ATI thresholds. Aside from those in $\chi_{1}^{(\mu, \nu)}$, additional TAs are hidden in the exact (beyond KA) coefficients $\phi_{\nu}$ (which satisfy equations involving matrix elements that have series expansions similar to those in Eq. (3) [13]). Although the singularities in $\phi_{\nu}$ are crucial for predicting TAs in ATI spectra [21], for HG our numerical analysis shows that for low $\omega$ and relatively high $F$, they have only a small effect compared to TAs in $\chi_{1}^{(\mu, \nu)}$. However, the KA fails for low intensities, when the atomic-potential-mediated interchannel coupling largely dominates the laser-induced coupling. For example, the LOPT result of Eqs. (2) and (3) for $n=3$ near the two-photon ATI threshold $(\omega \approx 1 / 2)$ is [22]

$$
\begin{aligned}
\chi_{1}= & \ell F^{3}\left(c^{(0)}+c^{(1)} \sqrt{2 \omega-1}\right), \\
\left|\widetilde{\mathbf{d}}_{3}\right|^{2}= & \ell^{2} F^{6}\left|c^{(0)}\right|^{2}-2 \ell^{2} F^{6} \sqrt{|2 \omega-1|} \\
& \times \begin{cases}\operatorname{Im}\left(c^{(0) *} c^{(1)}\right), & \omega>1 / 2, \\
\operatorname{Re}\left(c^{(0)} * c^{(1)}\right), & \omega<1 / 2 .\end{cases}
\end{aligned}
$$

The exact values of the $c^{(0,1)}$ coefficients are: $c^{(0)} \approx-7.56$ $-2.65 i ; \quad c^{(1)} \approx 3.10+1.31 i$. However, the KA calculation [using Eq. (5) in the LOPT analysis of Eqs. (2) and (3)] gives completely different results: $c^{(0)} \approx 21.81-3.02 i ; c^{(1)}=1 / 12$. 


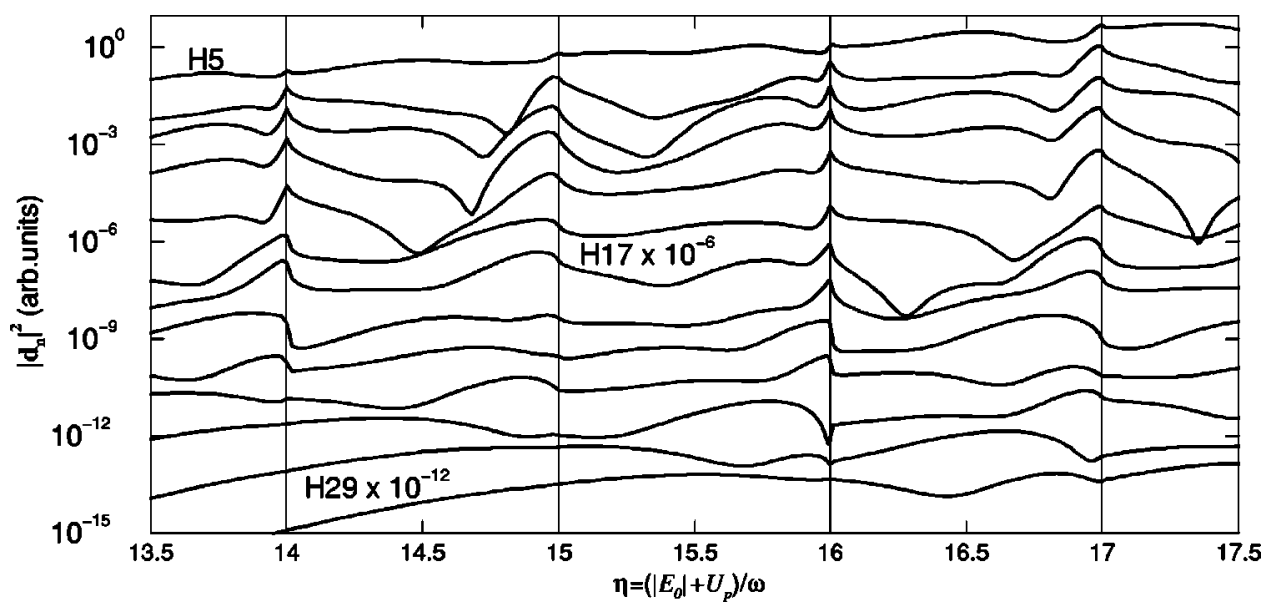

FIG. 1. $\left|\mathbf{d}_{n}\right|^{2}$ with $5 \leqslant n \leqslant 29$ for fixed frequency $\omega=0.1055$ and varying intensity $(0.097 \leqslant F \leqslant 0.137)$. The integers $n$, on the abscissa indicate the $n$-photon ionization thresholds. For better visualization, the curves are separated through multiplication by $10^{-N}$ with $N$ increasing from 0 (top, fifth harmonic) to 12 (bottom, 29th harmonic). On the scale of this plot, $\epsilon \approx E_{0}$.

This discrepancy occurs because as $F$ decreases, the TAs stemming from the $\phi_{\nu}$ coefficients become dominant compared with those of $\chi_{1}^{(\mu, \nu)}$.

For the more interesting case of low $\omega$ and relatively high $F$, we find, based on exact quantum calculations, that the KA [i.e., the use of Eq. (5) for $\phi_{\nu}$ ] gives good numerical results for $\mathrm{HG}$ and reproduces (within a few percent) the features visible in our Figs. 1 and 2 for the case of linear laser polarization, $\ell=1$. Our results on the intensity dependence of HG rates are in qualitative agreement with results of Ref. [7] for $n=9$; also the exact results in Figs. 1 and 2 confirm the CC-related origin of structures in HG spectra proposed in Ref. [7]. Similar structures were obtained also in Ref. [19] and were interpreted [9] as the result of interference between two "effective" electron trajectories. We agree that the stationary points contributing near the threshold $\eta=q$ may have a special interpretation; however, the stationary phase approximation, which links the quantum analysis to the quasiclassical one, cannot be carried out in the usual way exactly at a threshold since it involves a Taylor expansion, and, therefore, needs modification [9].

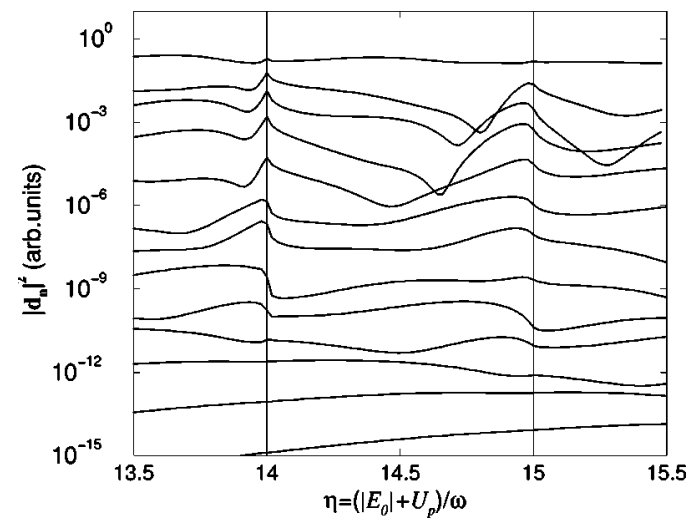

FIG. 2. Same as Fig. 1 but for fixed $F=0.103$ as $\omega$ varies $(0.1078 \geqslant \omega \geqslant 0.0992)$. $F$ is such that the 14 -photon channel closes for the same values of $F$ and $\omega$ as in Fig. 1.
Equation (3) suggests that if $F_{t h}$ and $\omega_{t h}$ are chosen such that $\operatorname{Re} \eta\left(F_{t h}, \omega_{t h}\right)=q$, then the only relevant parameter describing the behavior of any HG rate over some interval around the $q$ th threshold is the distance in energy from the threshold: $q-\operatorname{Re} \eta$ (i.e., $q \omega-U_{p}+\operatorname{Re} \epsilon$ ). In particular, scanning in energy across the threshold by either a change in $F$ near $F_{t h}$ or a change in $\omega$ over $\omega_{t h}$ yields the same behavior over the region of the $q$ th ATI threshold. This fact is illustrated in Figs. 1 and 2, where $\left|\widetilde{\mathbf{d}}_{n}\right|^{2}$ for the harmonics in the plateau region are plotted as functions of $\eta$. In Fig. 1 we keep $\omega$ fixed and vary $F$, whereas in Fig. 2 we fix $F$ and vary $\omega$. The intersection point of the two figures is the 14-photon $\mathrm{CC}$, which corresponds to $F_{t h}=0.103$ and $\omega_{t h}=0.1055$ in our scaled units. (For Ar, with $E_{0}=15.76 \mathrm{eV}$, this corre-

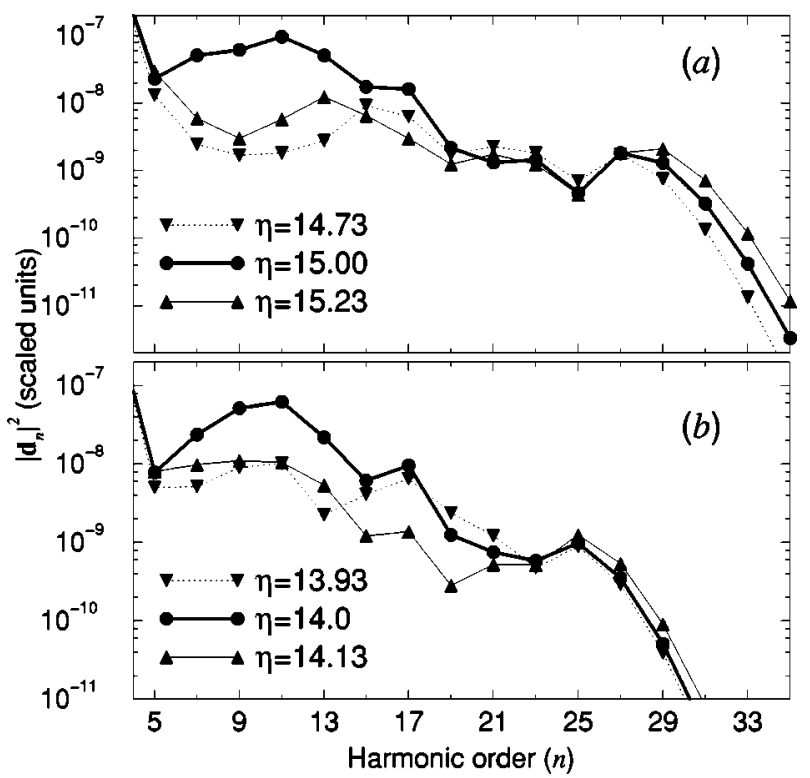

FIG. 3. Plateau part of the HG spectrum near the closings of the 15-photon (a) and 14-photon (b) ionization channels. The spectra are for fixed $\omega=0.1055$ and intensities below, at, and above the thresholds, as indicated by the figure legends. 
sponds to $\lambda \approx 750 \mathrm{~nm}$ and $I \approx 1.5 \times 10^{14} \mathrm{~W} / \mathrm{cm}^{2}$.) Note the exact correspondence of the features seen near $\eta=14$ in both figures over a range comparable to the distance to the neighboring thresholds. Note also the wider features appearing at odd CCs as opposed to the sharper ones appearing at even ones (which can be traced back to the odd/even photoelectron angular-momenta CCs contributing at the odd/even multiphoton thresholds). While very close to the threshold the dominant contribution is always that of the lowest angularmomentum component of the closing channel, on the energy interval considered here the nonperturbative strength of the interchannel couplings modifies this behavior. Note in Fig. 1 that the TAs vanish for harmonics near the plateau cutoff, in agreement with Refs. [7,20].

One practical importance of these findings is the possibility of controlling the HG process. Figure 3 shows HG spectra near one odd and one even CC. One sees that a variation of the laser intensity by less than $10 \%$ in the $\mathrm{CC}$ region results in a change of more than an order of magnitude in the HG rates across a major part of the plateau. As experimental techniques now permit observation of these features [11], small changes in $F$ or $\omega$ can be used to tune the harmonics. Alternatively, since enhancement of HG rates depends only on distance from a threshold, one can also control the threshold position, e.g., the same variation of $U_{p}(\approx 10 \%$ in our examples) necessary to scan the threshold-related HG maxima can be achieved using a second (infrared) laser with ten times smaller frequency and 100 times smaller intensity.

Most HG experiments are done with rare gases, in which the active electron experiences the long-range Coulomb po- tential (CP) of the core. CP specific TAs have the form of steplike jumps [23]. It is difficult to predict their exact effect on HG without a detailed study of the intense-laser-induced coupling of the channels. For HG at low intensities one finds typical CP peculiarities (see, e.g., Fig. 2.5 in Ref. [24] for $n=3 \mathrm{HG}$ in the $\mathrm{H}$ atom). In general, one expects a combination of Coulomb and short-range specific TAs with their relative importance determined by the atom considered. In experiments, all TAs will be broadened by the effects of finite pulses (but their observation is nevertheless possible [11]). Although the presence of excited states is an important difference between a ZRP and a CP, only the lower excited states may be distinctly observed as resonances. Higher members of the Rydberg series cannot be resolved close to thresholds and an accurate theoretical modeling leads to the step like jumps specific to the CP [23].

In summary, we have presented an exact quantum analysis of HG for a ZRP model. Our analytical and numerical results show that for this case the dynamics of the HG process and its dependence on laser parameters is essentially determined by the well-known threshold phenomena of multichannel reaction theory. We predict broad, CC-related, resonant like enhancements of HG rates in the plateau region. Our quantum analysis complements the existing quasiclassical picture of HG as resulting from interference between electron trajectories.

This work was supported by NSF Grant No. PHY0070980, by RFBR Grant No. 00-02-17843, and by Grant No. E00-3.2-515 of the Russian Ministry of Education.
[1] M. Protopapas et al., Rep. Prog. Phys. 60, 389 (1997).

[2] B.W. Shore and P.L. Knight, J. Phys. B 20, 413 (1987); J.H. Eberly et al., Phys. Rev. Lett. 62, 881 (1989); K.J. Schafer et al., ibid. 70, 1599 (1993).

[3] Ph. Balcou and A. L'Huillier, Phys. Rev. A 47, 1447 (1993).

[4] M.B. Gaarde and K.J. Schafer, Phys. Rev. A 64, 013820 (2001).

[5] J.F. Krause et al., Phys. Rev. A 45, 4998 (1992); R.M. Potvliege and P.H.G. Smith, in Super-Intense Laser-Atom Physics, edited by B. Piraux et al. (Plenum, New York, 1993), p. 173.

[6] A.I. Baz', Zh. Éksp. Teor. Fiz. 33, 923 (1957) [Sov. Phys. JETP 6, 709 (1958)].

[7] W. Becker et al., Phys. Rev. A 46, R5334 (1992).

[8] L.V. Keldysh, Zh. Éksp. Teor. Fiz. 47, 1945 (1964) [Sov. Phys. JETP 20, 1307 (1965)].

[9] M.Yu. Kuchiev and V.N. Ostrovsky, J. Phys. B 34, 405 (2001).

[10] M. Lewenstein et al., Phys. Rev. A 52, 4747 (1995).

[11] E.S. Toma et al., J. Phys. B 32, 5843 (1999).
[12] N.L. Manakov et al., Phys. Rep. 141, 319 (1986).

[13] N.L. Manakov and A.G. Fainshtein, Zh. Éksp. Teor. Fiz. 79, 751 (1980) [Sov. Phys. JETP 52, 382 (1980)].

[14] N.L. Manakov et al., J. Phys. B 33, R141 (2000).

[15] B. Borca et al., Phys. Rev. Lett. 85, 732 (2000); B. Borca et al. in Super-Intense Laser-Atom Physics edited by B. Piraux and K. Rzażewski (Kluwer, Dordrecht, The Netherlands, 2001), p. 249 .

[16] B. Borca et al., Phys. Rev. Lett. 87, 133001 (2001).

[17] W. Becker et al., Phys. Rev. A 56, 645 (1997).

[18] M. Lewenstein et al., Phys. Rev. A 49, 2117 (1994).

[19] M.Yu. Kuchiev and V.N. Ostrovsky, Phys. Rev. A 60, 3111 (1999).

[20] W. Becker et al., Phys. Rev. A 50, 1540 (1994).

[21] B. Borca et al., Phys. Rev. Lett. 88, 193001 (2002).

[22] For the full PT result for $\chi_{1}(3 \omega)$, see Eq. (2.112) of Ref. [24].

[23] See, e.g., R.G. Newton, Scattering Theory of Waves and Particles, 2nd ed. (Springer, New York, 1982), Chap. 17.

[24] A.G. Fainshtein et al., Phys. Rep. 210, 111 (1992). 